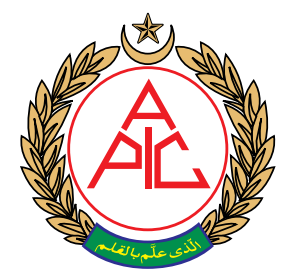

Department of Neurology, Sligo University Hospital, Sligo (Ireland) Salman Mansoor [salmanmansoor. dr@gmail.com]

Kevin Murphy [dkevinmurphy@gmail. com]

Siobhan Kelly [siobhankelly77@gmail. com]

Correspondence: Dr Salman

Mansoor,

Department of Neurology, The Mall, Rathquarter, Sligo, F91 H684, (Ireland) Phone: +353838281797; E-mail: salmanmansoor.dr@gmail.com

Received: 28 December 2019;

Reviewed: 10 January 2020;

Accepted: 10 January 2020

\section{Disulfiram induced bilateral foot drop; an under-recognised cause for peripheral neuropathy}

\author{
Salman Mansoor, Kevin Murphy, Siobhan Kelly
}

\begin{abstract}
Disulfiram is commonly used in chronic alcoholics as an effective treatment. We report a case of a 50 years old Irishman who was on disulfiram for the last nine years. He developed progressive bilateral foot drop, which started with one foot and later on progressed to bilateral involvement over a period of eight months. Our case report highlights the need to take into consideration disulfiram as one of the possible causes of peripheral neuropathy, which is a rare side effect of this drug.
\end{abstract}

Key words: Disulfiram; Foot drop; Peripheral neuropathy; Chronic alcoholism

Abbreviations: Abductor Digiti Minimi - ADM; Extensor Digitorum Brevis - EDB, Abductor Hallucis - AH; Antinuclear antibodies - ANA; Ribonucleoprotein - RNP; Cerebrospinal fluid - CSF; Non-recordable - NR; Electromyography - EMG; Flexor digitorum indicis - FDI; Sensory nerve action potential - SNAP

Citation: Mansoor S, Murphy K, Kelly S. Disulfiram induced bilateral foot drop; an under-recognised cause for peripheral neuropathy. Anaesth pain intensive care 2020;24(1):108-110. DOI: https://doi.org/10.35975/apic.v24i1.1234

\section{INTRODUCTION}

Disulfiram has been commonly marketed as an antabuse, and has been in practice for treating chronic alcoholism since 1948. ${ }^{1}$ The mode of effect is secondary to it's inhibitory effect on acetaldehyde dehydrogenase causing an immediate hangover effect when taken with alcohol even in small amounts. ${ }^{2}$ The usual mechanism for alcohol metabolism requires acetaldehyde dehydrogenase to breakdown acetaldehyde. ${ }^{2}$ Disulfiram thus builds acetaldehyde causing its unpleasant effects if taken in conjunction with alcohol. The side effects of disulfiram are mainly involve central nervous system but it has also rarely been reported to cause peripheral neuropathy. ${ }^{2}$

We present this case report to stress the need of keeping disufiram in mind while dealing a patient of peripheral neuropathy, particularly in an alcohol indulged locality or society.

\section{CASE REPORT}

We report a case of a 50 year old male patient from the north west of Ireland. He had serious alcohol issues for the last 10 years. He presented to the neurology outpatient department with an eight month history of progressive weakness in lifting his feet. His symptoms started almost eight months ago when he started noticing difficulty in his walk due to his left foot weakness. Over the course of next few months his weakness became more pronounced. Subsequently he also started noticing a similar pattern of weakness in his right foot with difficulty in lifting it. He started walking with a single crutch, which later rendered him dependent on using two crutches for even a small distance. He had been severely restricted in his daily activities due to this weakness. There were no bowel or bladder symptoms and he denied any weakness of his arms and hands.

His background medical history included chronic alcohol abuse 10 years ago, depression, chronic obstructive pulmonary disease, epilepsy and an old left caudate ischemic stroke. His drug history included disulfiram $400 \mathrm{mg} \mathrm{OD}$, lamotrigine $100 \mathrm{mg}$ $\mathrm{BD}$, aspirin $75 \mathrm{mg} \mathrm{OD}$, bisoprolol $2.5 \mathrm{mg}$, atorvastatin $40 \mathrm{mg}$ OD, and Quetiapine $25 \mathrm{mg}$ TDS.

He had been taking disulfiram for the last 9 years, which significantly helped him in coping with his alcohol problem. About one year back his general practitioner tried to stop his disulfiram, but after stopping it for few days he resumed it again as he felt better on it.

His physical examination showed bilateral foot drop and he walked with the help of a pair of cruthes with a high-stepping gait. Romberg's test was positive on eye 
closure. His power in both feet was $1 / 5$ on dorsiflexion, and eversion plantar flexion was graded to be $4 / 5$. Deep tendon reflexes were $2+$ in his knees while absent in his ankles with down-going bilateral plantar responses. There was no weakness in his proximal leg or hip muscles. The power in his hands and arms was normal. There were decreased touch and vibration senses up to his ankles.

\section{Investigations:}

$\mathrm{He}$ had an extensive battery of tests that included full blood count, ESR, CRP, HbAlC, blood glucose creatinine kinase, electrolytes, renal functions, thyroid functions, liver function tests, vitamin B12 level, folate, serum angiotensin converting enzyme, calcium, copper and zinc levels which were all within normal limits.

His autoimmune profile for ANA, Anti-U1, antiRNP, anti-Ro, anti-La, anti-SCL70, anti-Jol, anti-centromere and antiganglioside were negative. Serum protein electrophoresis didn't show any para-protein bands on immuno-fixation. Copper and zinc levels checked were within normal ranges. Paraneoplastic antibodies anti-Hu/Yo/Ri/CV2/ amphiphysin/Ma2/myelin associated glycoproteins were negative. Cerebrospinal fluid (CSF) analysis showed normal cell count, protein and CSF glucose.

Left Tibial Knee -AH
Table 1: Sensory nerve conduction study

\begin{tabular}{l|l|c|c|c|c}
\multicolumn{1}{c|}{ Nerve/Sites } & \multicolumn{1}{c|}{ Recording Site } & $\begin{array}{c}\text { Peak Latency } \\
\text { (ms) }\end{array}$ & $\begin{array}{c}\text { Amplitude } \\
(\mathbf{u V})\end{array}$ & $\begin{array}{c}\text { Distance } \\
(\mathbf{c m})\end{array}$ & $\begin{array}{c}\text { Velocity } \\
\text { (m/s) }\end{array}$ \\
\hline Right Sural / Calf & Lateral Malleolus & 5.63 & 6.7 & 14 & 24.9 \\
\hline Left Sural / Calf & Lateral Malleolus & 5.16 & 4.4 & 14 & 27.2 \\
\hline Left Radial / Forearm & Wrist & 2.76 & 19.5 & 10 & 36.2 \\
\hline
\end{tabular}

Table 2: Motor nerve conduction study

\begin{tabular}{|c|c|c|c|c|c|c|c|}
\hline Nerve/Sites & $\begin{array}{l}\text { Rec. } \\
\text { Site }\end{array}$ & $\begin{array}{l}\text { Latency } \\
\text { (ms) }\end{array}$ & $\begin{array}{c}\text { Amplitude } \\
\text { (mV) }\end{array}$ & $\begin{array}{c}\text { Relative } \\
\text { Amplitude \% }\end{array}$ & $\begin{array}{c}\text { Area } \\
(\mathrm{mV} \mathrm{ms})\end{array}$ & $\begin{array}{c}\text { Distance } \\
\text { (cm) }\end{array}$ & $\begin{array}{c}\text { Velocity } \\
(\mathrm{m} / \mathrm{s})\end{array}$ \\
\hline \multicolumn{8}{|c|}{ Left Ulnar- Abductor Digiti Minimi (ADM) } \\
\hline Wrist & ADM & 3.07 & 12.5 & 100 & 36.3 & 7 & \\
\hline B. Elbow & ADM & 7.19 & 9.8 & 78.8 & 29.5 & 19 & 46.2 \\
\hline A.Elbow & ADM & 8.75 & 7.6 & 61.2 & 23.2 & 7 & 44.4 \\
\hline
\end{tabular}

Right Common Peroneal Extensor Digitorum Brevis (EDB)

\begin{tabular}{l|l|l|l|l|l|l|l}
\hline Ankle & EDB & NR & NR & NR & NR & 8 & \\
\hline Fib. Head & EDB & NR & NR & NR & NR & 27 & NR \\
\hline Knee & EDB & NR & NR & NR & NR & 7 & NR \\
\hline
\end{tabular}

Left Common Peroneal - EDB

\begin{tabular}{l|l|l|l|l|l|l|l}
\hline Ankle & EDB & NR & NR & NR & NR & 8 & \\
\hline Fib. Head & EDB & NR & NR & NR & NR & 30 & NR \\
\hline Knee & EDB & NR & NR & NR & NR & 7 & NR \\
\hline
\end{tabular}

Right Tibial Knee -Abductor Hallucis (AH)

\begin{tabular}{l|l|l|l|l|l|l|l}
\hline Ankle & AH & NR & NR & NR & NR & 8 & \\
\hline Knee & AH & NR & NR & NR & NR & 37 & NR \\
\hline
\end{tabular}

\begin{tabular}{l|l|c|c|c|c|c|c}
\hline Ankle & AH & 10.78 & 0.1 & 100 & 0.2 & 8 & \\
\hline Knee & AH & NR & NR & NR & NR & 37 & NR \\
\hline
\end{tabular}

\section{Nerve conduction studies:}

Nerve conduction studies showed non-recordable (NR) potentials in his both lower limbs in motor study, with normal nerve potentials in his upper limbs as shown in Table 1 and Table 2.

\section{Electromyography (EMG):}

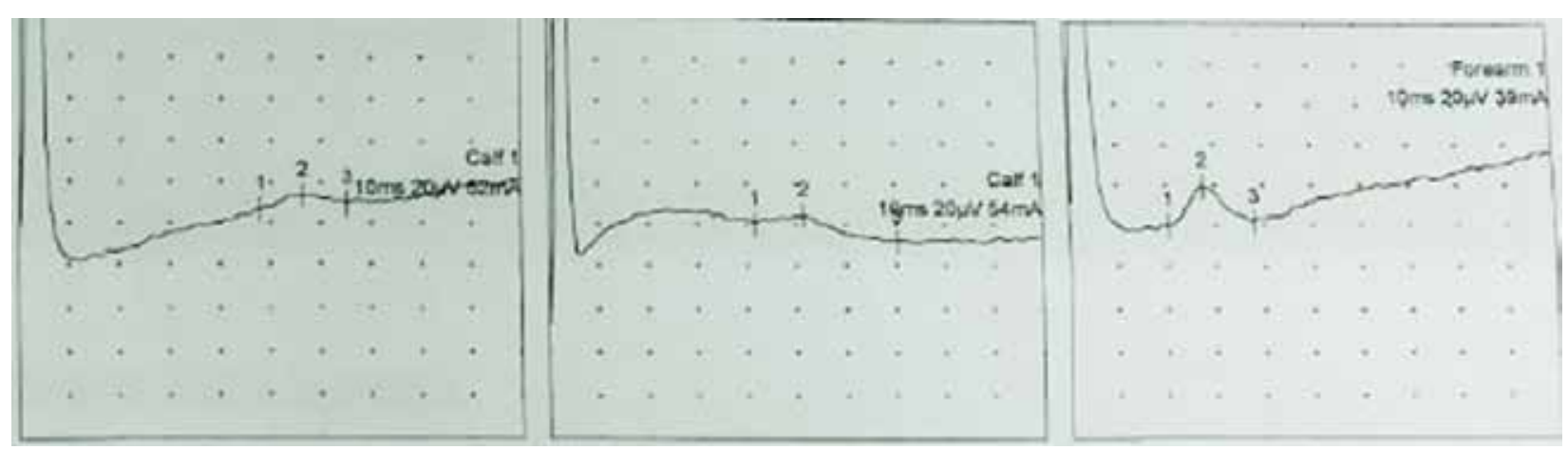

Figure 1: Showing the waveforms which were recorded during sensory nerve conduction study 
Concentric needle EMG showed bilateral medial gastrocnemii and tibialis anterior muscles showing increased insertional activity with fibrillation potentials and positive sharp waves. There were chronic reinnervation changes seen in all leg muscles studied. Left arm muscles e.g. biceps and flexor digitorum indicis (FDI) were studied and found normal.

\section{Outcome:}

Based on these findings diagnosis of disulfiram related peripheral neuropathy was made. His disulfiram was stopped and he was started on long term rehabilitation. At this point it's unclear whether he is going to regain any strength with physiotherapy back or not.

\section{DISCUSSION}

Disulfiram neuropathy has been described as a distal axonopathy related to the "dying-back" effect of axonal degeneration. ${ }^{3}$ Carbon disulfide is thought to cause peripheral neuropathy as a break down product of disulfiram metabolism. ${ }^{4}$ The relative sparing of sensory nerve action potentials (SNAPs) suggest preservation of large fibre sensory nerves in our case. The severity and prognosis of disulfiram associated neuropathy has been reported to correlate with the dose and duration of therapy, various co-morbidities, and rehabilitation. ${ }^{5}$

\section{CONCLUSION}

Peripheral neuropathy due to disulfiram is an under recognised cause for peripheral neuropathy. The diagnostic limitation lies in unavailability of any specific test to establish this rare complication. Most of these patients are heavy drinkers for years which makes odds to the diagnostic uncertainty. The workup is mainly to exclude other causes. Peripheral neuropathy is a preventable complication which the practicing clinician should be more aware about in patients who are on disulfiram.

Conflict of interest: None declared by the authors

Authors' contribution: All authors were involved in diagnosing and treating the patient as well as writing of this manuscript

\section{REFERENCES}

1. Johan Edman: A_Medical Challenge: The Alcohol Disease in Sweden 1946-1955. Social History of Medicine. February 2020;33(1):22446 [Free full text] DOI: https://doi. org/10.1093/shm/hky050

2. Tran AT, Rison RA, Beydoun SR. Disulfiram neuropathy: two case reports. J Med Case Rep. 2016 Mar 31;10:72. [PubMed] [Free full text] DOI: 10.1186/s13256-016-0865-z
3. Filosto $M$, Tentorio $M$, Broglio L, Buzio S, Lazzarini C, Pasolini $M P$, et al. Disulfiram neuropathy: two cases of distal axonopathy. Clin Toxicol. 2008;46:314-6. [PubMed] [Free full text] DOI: 10.1080/15563650701636390

4. Kulkarni RR, Pradeep AV, Bairy BK. Disulfiram-induced combined irreversible optic neuropathy and reversible peripheral neuropathy: a prospective case report and review of the literature. J Neuropsychiatry Clin Neurosci. 2013;25:339-42. [PubMed] [Free full text] DOI: 10.1176/appi. neuropsych.12120410

5. Rossum JV, Roos RC, Bots GT. Disulfiram polyneuropathy. Clin Neurol Neurosurg. 1984;86-2:81-7. [PubMed] DOI: $\underline{10.1016 / 0303-}$ 8467(84)90070-2 